\title{
Inverse cascade suppression and shear layer formation in MHD turbulence subject to a guide field and misaligned rotation
}

\author{
Santiago J. Benavides ${ }^{1} \dagger$, Keaton J. Burns ${ }^{2,3}$, Basile Gallet ${ }^{4}$, \\ James Y-K. Cho ${ }^{3}$ and Glenn R. Flierl ${ }^{1}$ \\ ${ }^{1}$ Department of Earth, Atmospheric, and Planetary Sciences, Massachusetts Institute of \\ Technology, Cambridge, MA 02139, USA \\ ${ }^{2}$ Department of Mathematics, Massachusetts Institute of Technology, Cambridge, MA 02139, \\ USA \\ ${ }^{3}$ Center for Computational Astrophysics, Flatiron Institute, New York, NY 10010, USA \\ ${ }^{4}$ Université Paris-Saclay, CNRS, CEA, Service de Physique de l'Etat Condensé, 91191 \\ Gif-sur-Yvette, France
}

(Received $\mathrm{xx}$; revised $\mathrm{xx}$; accepted $\mathrm{xx}$ )

\begin{abstract}
Astrophysical flows are often subject to both rotation and large-scale background magnetic fields. Individually, each is known to two-dimensionalize the flow in the perpendicular plane. In realistic settings, both of these effects are simultaneously present and, importantly, need not be aligned. In this work, we numerically investigate threedimensional forced magnetohydrodynamic (MHD) turbulence subject to the competing effects of global rotation and a perpendicular background magnetic field. We focus on the case of a strong background field and find that increasing the rotation rate from zero produces significant changes in the structure of the turbulent flow. Starting with a twodimensional inverse energy cascade at zero rotation, the flow first transitions to a forward cascade of kinetic energy, then to a shear-layer dominated regime, and finally to a second shear-layer regime where the kinetic energy flux is strongly suppressed and the energy transfer is mediated by the induced magnetic field. We show that the first two transitions occur at distinct values of the Rossby number, and the third occurs at a distinct value of the Lehnert number. The three-dimensional results are confirmed using an asymptotic two-dimensional, three-component model, which allows us to extend our results to the planetary-relevant case of an arbitrary angle between the rotation vector and guide field. More generally, our results demonstrate that, when considering the simultaneous limits of strong rotation and a strong guide field, the order in which those limits are taken matters in the misaligned case.
\end{abstract}

\section{Introduction}

Turbulence in geophysical and astrophysical settings contains additional physical ingredients that break the isotropy of the flow, a traditional assumption in classical turbulence theory, thereby adding complexity to the system at hand (Frisch 1995; Davidson 2013; Alexakis \& Biferale 2018). This includes, but is not limited to, rotating, electrically conducting, stratified, and large aspect ratio systems. Asymptotic regimes are sought out to simplify the system, thus allowing previous ideas and techniques of idealized turbulence to be used. This is done by studying the limiting equations as a control parameter (rotation, aspect ratio, etc.) is taken to zero or infinity. For example, one particular success is the

$\dagger$ Email address for correspondence: santib@mit.edu 
quasigeostrophic approximation, which predicts horizontal motion in the presence of stratification and rapid rotation (Charney 1971; Vallis 2017). More generally, in rapidly rotating systems without stratification and with periodic boundary conditions, the flow becomes two-dimensional (2D), invariant along the rotation axis (Smith \& Waleffe 1999; Mininni \& Pouquet 2010; Gallet 2015; Buzzicotti et al. 2018). A similar simplification occurs in plasmas in the presence of a strong uniform background magnetic 'guiding' field, reducing the dynamics to 2D magnetohydrodynamics (MHD) (Montgomerv \& Turner 1981; Nazarenko 2007; Bigot \& Galtier 2011; Alexakis 2011; Sujovolsky \& Mininni 2016), and further to 2D hydrodynamics (HD) if the magnetic field is not forced (Alexakis 2011; Sujovolsky \& Mininni 2016). Both of these limits produce 2D HD turbulence, which is characterized by the presence of an inverse cascade of energy, in which energy goes from the forcing scale towards larger scales (Kraichnan 1967; Boffetta \& Ecke 2012; Alexakis \& Biferale 2018). This is in contrast to the forward energy cascades found in 3D HD and MHD turbulence in the absence of a guiding field, where energy cascades to smaller scales. The asymptotic regimes allow one to use energy cascade arguments to help understand turbulent geophysical and astrophysical phenomena. For example, the inverse cascade in the quasigeostrophic system is thought to contribute to the formation of jets in rapidly rotating planetary atmospheres (Rhines 1975; Held \& Larichev 1996; Cho \& Polvani 1996a, $b$; Arbic \& Flierl 2004; Tobias et al. 2007; Gallet \& Ferrari 2021). An analogous cascade mechanism is thought to be responsible for the formation of poloidal jets in tokamak plasmas in the presence of a strong background toroidal guiding magnetic field (Diamond et al. 2005).

In many geophysical and astrophysical contexts, however, it is expected that a fluid is subject to some combination of rotation, magnetic field, and stratification (Cho 2008; Davidson 2013; Vallis 2017). Asymptotic analysis of these combined cases is more difficult, where often the order in which the limits are taken matters, and knowing which regime is observed in nature (and how the energy cascades behave) is a challenge (Aurnou et al. 2015). Furthermore, real physical systems are not subject to infinite rotation rates or infinite background magnetic field strengths and reality often lies at intermediate values. There is currently no existing theory for the cascade direction of such intermediate parameters, and it is only more recently through state-of-the-art simulations (Smith et al. 1996; Smith \& Waleffe 1999; Celani et al. 2010; Pouquet \& Marino 2013; Deusebio et al. 2014; Marino et al. 2015) and lab experiments (Xia et al. 2011; Campagne et al. 2014; Baker et al. 2018) that we are able to carefully investigate their turbulent dynamics. These studies looking into the cascade of conserved quantities in geophysical and astrophysical flows have revealed the presence of bidirectional cascades $\oplus$ at intermediate parameter values, in which a fraction of the conserved quantity input by the forcing goes to large scales whereas the rest goes to small scales Alexakis \& Biferale 2018; Pouquet et al. 2019). Most of these systems seem to form bidirectional cascades at particular critical values of the control parameters. Numerical simulations are crucial in revealing the behavior of turbulent systems in configurations and parameter values that are out of reach of asymptotic methods.

Here we investigate the turbulent dynamics of an incompressible electrically-conducting MHD fluid subject to rotation and a misaligned uniform background magnetic field using a series of direct numerical simulations. Such a configuration is expected to represent the turbulent dynamics in the atmospheric interiors of gas giant planets in the transition

$\dagger$ Not to be confused with dual cascade scenarios, where the system has two conserved quadratic quantities which cascade in different directions, such as in 2D HD turbulence with the forward cascade of enstrophy and inverse cascade of energy. 
region between the outer, neutral atmosphere and the deep, ionized one (e.g., Liu et al. (2008); Dietrich \& Jones (2018); Benavides \& Flierl (2020)). There, the dynamics are characterized by rapid rotation and the presence of a strong background field generated by the dynamo in the deep interior region below. A simplified case of a dipole magnetic field present in the transition layer would suggest that the alignment between rotation and the background field would vary with latitude. The latest Juno measurements by Moore et al. (2018) show, however, that the background field around the transition region is quite 'patchy', but we still expect the misalignment with rotation to be a generic feature. In these regions the electrical conductivity is expected to be quite low (Liu et al. 2008; French et al. 2012; Dietrich \& Jones 2018). For the sake of generality, in the following we investigate a model with rather large conductivity, before discussing how most of the results carry over to the low-conducting case in Section 5. To some extent, the ultimate effect of the background magnetic field is the same, resulting in anisotropic flows, and eventually the two-dimensionalization of the flow perpendicular to the field (Sommeria \& Moreau 1982; Vorobev et al. 2005; Thess \& Zikanov 2007; Favier et al. 2010; Gallet \& Doering 2015; Baker et al. 2018). While our interests are at the fundamental level, with application to gas giant planets in mind, the effects of a background field and (possibly misaligned) rotation also need to be considered in the formation and dynamics of ionized protoplanetary disks in the presence of the host star's magnetosphere (Fromang 2005; Armitage 2011; Joos et al.|2012; Simon et al. 2013, 2018). Both of the astrophysical settings mentioned so far are geometrically confined, so we will not explore large domain size effects in this work (see discussion in Section 4).

More generally, given the prevalence of astrophysical systems which are both ionized and undergoing rotation, we expect our results to be general enough to apply in other contexts. Our idealized system has simplified forcing and boundary conditions compared to realistic astrophysical settings. However, its role is to uncover the dynamics of the small scales, which can eventually guide parametrizations of sub-grid scale fluxes in large-scale models of astrophysical objects.

In particular, we are interested in understanding what happens when there are two, two-dimensionalizing effects which act in different directions. What is the fate of the inverse cascade and how 'fragile' is it to the variation in the secondary control parameter? Focusing on the case of a strong background field, we find that increasing the rotation rate from zero produces significant changes in the structure of the turbulent flow. Starting from a two-dimensional inverse cascade scenario at zero rotation, we find four distinct dynamical regimes as we increase rotation: for weak rotation rates we observe a bidirectional cascade of kinetic energy, with energy flux to large scales decreasing as rotation is increased, and negligible induced magnetic energy. For rotation rates past some critical point, the flow transitions to a purely forward cascade of kinetic energy. Further increasing the rotation rate results in a shear-layer dominated regime, where nonlinearities at large scales are suppressed. Finally, at the largest rotation rates we investigated, we found a second shear-layer regime where the induced magnetic energy is no longer negligible, the kinetic energy flux is strongly suppressed, and the energy transfer is purely mediated by nonlinear terms which include the induced magnetic field. Using a two-dimensional, three-component asymptotic model of our system, we also show that the first three regimes are separated by sharp transitions, hinting at the existence of a bifurcation in the behavior of the turbulent flow. One is found to be similar to other previously-found transitions from a bidirectional cascade to a forward one, while the other shows subcritical behavior including a discontinuity in the order parameter and hysteresis. The transition to the magnetically active regime is beyond the scope of the reduced model, but we show that it also sharpens towards a critical value as 
the background magnetic field strength increases. We find more generally that, when considering the limit of strong rotation and strong magnetic field, the order in which those limits are taken matters.

In section 2 we introduce the system we will study: rotating MHD in the presence of a background magnetic field, referred to as $B \Omega$-MHD (Menu et al. 2019). In section 3 we discuss results from three-dimensional simulations in which the background magnetic field is strong and as we vary the rotation rate in a perpendicular direction. In section 4 we introduce a two-dimensional, three-component (2D3C) asymptotic model (similar to that derived in Montgomery \& Turner (1981)) representing the strong background magnetic field limit and including rotation, and discuss results from the simulations of that reduced system. Discussion and implications of our results are presented in section 5. where we extend our results to an arbitrary angle between rotation and background magnetic field, before discussing the low conductivity limit, relevant to planetary settings.

\section{Rotating MHD in the presence of a background magnetic field}

The equations for rotating magnetohydrodynamics in the presence of a uniform background magnetic field are (Shebalin 2006; Galtier 2014):

$$
\begin{aligned}
& \frac{\partial \boldsymbol{v}}{\partial t}+(\boldsymbol{v} \cdot \nabla) \boldsymbol{v}=-\nabla p^{*}-2 \boldsymbol{\Omega} \times \boldsymbol{v}+(\nabla \times \boldsymbol{b}) \times\left(\boldsymbol{B}_{\mathbf{0}}+\boldsymbol{b}\right)+\boldsymbol{D}_{v}+\boldsymbol{f} \\
& \frac{\partial \boldsymbol{b}}{\partial t}+(\boldsymbol{v} \cdot \nabla) \boldsymbol{b}=\left(\boldsymbol{B}_{\mathbf{0}} \cdot \nabla\right) \boldsymbol{v}+(\boldsymbol{b} \cdot \nabla) \boldsymbol{v}+\boldsymbol{D}_{b} \\
& \nabla \cdot \boldsymbol{v}=0, \quad \nabla \cdot \boldsymbol{b}=0
\end{aligned}
$$

where $\boldsymbol{v}=\left(v_{x}, v_{y}, v_{z}\right)$ is the velocity field and $\boldsymbol{b}$ is the induced magnetic field, making up the two dynamical variables in this system. The two control parameters are $\boldsymbol{\Omega}$, the global rotation vector (with magnitude $\Omega$ ), and $\boldsymbol{B}_{\mathbf{0}}$, the uniform background field (with magnitude $B_{0}$ ). Other definitions include the total pressure modified by rotation $p^{*}$, which is normalized by the constant density $\rho_{0}$, and the dissipation terms, $\boldsymbol{D}_{v}$ and $\boldsymbol{D}_{b}$, which could be regular viscosity and magnetic diffusion, respectively, but might also include other forms of dissipation such as drag or hypodiffusion. The exact form of these terms will be described in Section 3, when the simulations are introduced. Magnetic fields are in Alfvén units, being normalized by $\sqrt{\rho_{0} \mu_{0}}$, where $\mu_{0}$ is the magnetic permeability. Finally, $\boldsymbol{f}$ is a body force, which will be used to inject energy into the velocity field.

The inviscid and perfectly conducting system conserves the total energy,

$$
E=\frac{1}{2} \int\left(\boldsymbol{v}^{2}+\boldsymbol{b}^{2}\right) d^{3} x
$$

However, when $\boldsymbol{\Omega}$ and $\boldsymbol{B}_{\mathbf{0}}$ are collinear, this system also conserves what's known as the parallel-helicity (Shebalin 2006) or hybrid-helicity (Galtier 2014; Menu et al. 2019). The collinear system has received considerable attention - favored over the misaligned case in part due to its extra conserved quantity and the potential relevance of its cascade for dynamo action(Shebalin 2006; Menu et al. 2019). It also possesses simplified linear wave solutions which have been used to develop a weak wave turbulence theory (Galtier 2014; Bell \& Nazarenko 2019). Here we will not be considering the collinear case, and so only the total energy will be conserved in our study of $B \Omega$-MHD in section 3 . Although waves are certainly present in our system, our work concerns the strongly turbulent dynamics of energy cascades (present partly in the zero frequency modes of the system). See Appendix A for the dispersion relation of waves in the misaligned case.

Most studies, with rotation and background magnetic field aligned or not, have focused 
on how rotation and a moderate background field affect the decay of kinetic and magnetic energies in unforced simulations (Lehnert 1955; Favier et al. 2012; Bell \& Nazarenko 2019; Baklouti et al. 2019). Menu et al. (2019) investigated the sensitivity of the cascade of hybrid helicity for various rotation and guide field alignments in forced-dissipative simulations. We consider the effects of rotation and a misaligned background magnetic field on the two-dimensionalization of the flow and the energy cascade, including the limits of strong rotation and strong background magnetic field.

In our study, the rotation and background magnetic field vectors are perpendicular to each other, namely, we have chosen $\boldsymbol{\Omega}=\Omega \hat{\boldsymbol{z}}$ and $\boldsymbol{B}_{\mathbf{0}}=B_{0} \hat{\boldsymbol{x}}$, the extension to an arbitrary angle between $\boldsymbol{\Omega}$ and $\boldsymbol{B}_{\mathbf{0}}$ being discussed in Section 5 . The turbulence is maintained at a statistically steady state by a forcing which inputs energy at a mean rate $I$ at a lengthscale $1 / k_{f}$ (see details in section 3). As a result, there is an emergent velocity scale $U$ defined to be $U^{3} \equiv I k_{f}^{-1}$, that we compare to the background field as a measure of its strength, the inverse Alfvén Mach number:

$$
M^{-1} \equiv \frac{B_{0}}{U}
$$

This dimensionless number can also be thought of as a measure of how the third term on the right hand side of equation (2.1) (the Lorentz force) and the first term on the right hand side of equation (2.2) compare to the advection terms in each respective equation, which would determine whether or not the background field affects the dominant dynamics. When $M^{-1} \gg 1$ the Lorentz force acts to constrain the velocity and induced magnetic fields so that they don't vary along the $x$-direction and most of the energy lies in the $k_{x}=0$ modes, resulting in 2D MHD (Montgomerv \& Turner 1981; Nazarenko 2007; Bigot \& Galtier 2011; Alexakis 2011; Sujovolsky \& Mininni 2016). It is important to note that while the dynamics depend on $y, z$, and $t$, all vector components can be non-zero in periodic domains. This is called two-dimensional, three-component (2D3C) dynamics Biferale et al. 2017). If the induced magnetic field isn't directly forced (as is the case in our study), this results in 2D3C HD and an inverse cascade of horizontal kinetic energy (Alexakis 2011; Sujovolsky \& Mininni 2016). All of our simulations lie in the regime of strong background magnetic field, $M^{-1} \gg 1$, making the rotation rate the main control parameter in our study. Does this asymptotic regime survive in the presence of rotation?

The relative strength of rotation is measured by the inverse Rossby number:

$$
R o^{-1} \equiv \frac{2 \Omega}{k_{f} U}
$$

This number measures the relative importance of the second term on the right hand side of equation (2.1) (the Coriolis force) to the advection term, which would determine whether or not the rotation affects the dynamics. Unlike the background magnetic field, the Coriolis force only directly affects the velocity field. For non-stratified rapidly rotating hydrodynamics in the absence of any magnetic field, $R o^{-1} \gg 1$, the strong Coriolis force acts to constrain the flow such that it doesn't vary along the $z$-direction and most of the energy lies in the $k_{z}=0$ modes (Smith \& Waleffe 1999; Mininni \& Pouquet 2010; Gallet 2015; Vallis 2017; Buzzicotti et al. 2018), which results in 2D3C HD where the dynamics depend only on $x, y$, and $t$. If the fluid is ionized and initialized with a non-zero seed magnetic field, rapid rotation doesn't necessarily result in 2D3C HD because there is no direct constraint on the induced magnetic field. Instead, if the transverse velocity component does not vanish, rapidly rotating dynamos are formed with $z$-dependent induced magnetic fields (Otani 1993; Smith \& Tobias 2004; Aurnou et al. 
2015; Seshasavanan \& Alexakis 2016b; Seshasavanan et al. 2017; Tobias 2021). However, since our base state is the $x$-independent 2D3C HD regime found when $M^{-1} \gg 1$, rapid rotation is expected to act to constrain the flow and prevent it from varying in the $z$ direction. Note that, in this configuration, rotation is in the plane of the $2 \mathrm{D}$ dynamics, not out of the plane as is often the case when it itself is the cause of the bidimensionalization. Since rotation is now in the plane of the two-dimensional velocities, the Coriolis force is expected to deflect horizontal velocities out of the plane, as will be discussed in section 4 when we introduce a reduced model for this system following Montgomery \& Turner (1981).

Our goal in this study is to investigate the effects that in-plane rotation has on the two-dimensional flow caused by a strong background magnetic field. In the next section we will describe results from direct numerical simulations of the $B \Omega$-MHD system for various rotation rates, paying particular attention to the resulting energy cascade and morphology of the flow field.

\section{Strong background field limit: 3D $B \Omega$-MHD simulations}

Equations (2.1)-(2.3) were solved numerically in a triply-periodic domain of side length $2 \pi L$ using the Geophysical High-Order Suite for Turbulence (GHOST) code (Mininni et al. 2011). The dissipation terms, $\boldsymbol{D}_{v}$ and $\boldsymbol{D}_{b}$, each consist of a 'hyperviscosity' and a large-scale dissipation term called 'hypoviscosity'. The hyperviscosity replaces the regular viscous and magnetic diffusion terms with a Laplacian of a higher order, in our case $\nabla^{2} \rightarrow-\nabla^{4}$. This higher order allows for the possibility of forcing at smaller lengthscales while still properly resolving the smallest scales at moderate resolutions. As long as the order of the Laplacian is not very large, hyperviscosity has been shown to have no significant effect on the turbulent properties of $3 \mathrm{D}$ turbulence, and we expect the same to be the case for our work (Agrawal et al. 2020). The hypoviscosity, which would appear as $\nu_{-} \nabla^{-2} \boldsymbol{v}$ on the right hand side of equation (2.1) and as $\eta_{-} \nabla^{-2} \boldsymbol{b}$ on the right hand side of equation (2.2), acts as a large-scale dissipation term. The resulting expressions for $\boldsymbol{D}_{v}$ and $\boldsymbol{D}_{b}$ are,

$$
\begin{aligned}
& \boldsymbol{D}_{v}=-\nu \nabla^{4} \boldsymbol{v}+\nu_{-} \nabla^{-2} \boldsymbol{v}, \\
& \boldsymbol{D}_{b}=-\eta \nabla^{4} \boldsymbol{b}+\eta_{-} \nabla^{-2} \boldsymbol{b},
\end{aligned}
$$

where $\nu$ is the kinematic 'hyper'-viscosity, $\eta=\left(\mu_{0} \sigma\right)^{-1}$ is the magnetic 'hyper'-diffusivity, $\sigma$ is the electrical conductivity, $\nu_{-}$is the 'hypo'-viscosity, and $\eta_{-}$is the magnetic 'hypo'diffusivity. Should an inverse cascade of a conserved quantity occur, this term ensures that no condensate forms, which would otherwise affect the cascades and inertial ranges (Chertkov et al. 2007; Xia et al. 2008; Gallet \& Young 2013; Seshasavanan \& Alexakis 2018; Alexakis \& Biferale 2018; van Kan \& Alexakis 2019). This is done by choosing the coefficients $\nu_{-}$and $\eta_{-}$such that the kinetic and magnetic energy at the largest scales is smaller than that of the next largest scales. The modified GHOST code which includes these alternative dissipative terms can be found in a public Github repository (Benavides 2019). It is a standard parallel pseudo-spectral code with a fourth-order Runge-Kutta scheme for time integration and a two-thirds dealiasing rule. The numerical model is nondimensionalized by $L$ and the forcing amplitude $f_{0}$, so that the wavenumbers $\boldsymbol{k}$ correspond to mode numbers of the domain and the forcing amplitude is one. The threedimensional forcing $\boldsymbol{f}$ is isotropic and constant in time, comprising of a summation of cosines with wavenumbers between $8<|\boldsymbol{k}|<10$, making $k_{f}=9$, and random phases. The forcing wavenumber range is chosen in an attempt to properly resolve both an 
inverse cascade and a forward cascade. $I \equiv\langle\boldsymbol{f} \cdot \boldsymbol{v}\rangle$ is the space- and time-averaged energy injection rate, where $\langle\cdot\rangle$ represents a space- and time-average. We do not force the induced magnetic field.

All runs, unless otherwise stated, are in the large background field regime with $M^{-1} \approx$ 84. We find this value to be large enough to produce the expected two-dimensionalization in the absence of rotation (Figure $1(a)$ ). Larger background magnetic field values result in significant restrictions in the time-step which would limit our ability to perform the same parameter sweep. The Reynolds and magnetic Reynolds numbers, defined, respectively, as $R e \equiv U / k_{f}^{3} \nu$ and $R e_{m} \equiv U / k_{f}^{3} \eta$ when considering hyperviscous and hyperdiffusive terms as we do, measure the relative strength of the advection terms compared to the hyperviscous and magnetic hyperdiffusion terms. For the simulations we performed, the Reynolds and magnetic Reynolds numbers were large (approximately 300) and equal to each other, i.e. the magnetic Prandtl number is set to one. We performed 14 runs at $M^{-1} \approx 84$ but at different values of $R o^{-1}$, ranging from $R o^{-1}=0$ to $R o^{-1}=27$. All averages and snapshots were taken in statistically steady states. See Table 1 for details of the simulations and a description of how we measured the nondimensional numbers.

In this study, we are partly concerned with the behavior of the energy cascade as rotation is varied. We expect the presence of a bidirectional cascade, where a fraction of the energy input by the forcing goes to large scales and the rest goes to small scales. As such, we define a measure for the fraction of energy that goes to large scales in the form of kinetic energy, $\varepsilon_{-}$, and that which goes to small scales in the form of kinetic energy $\varepsilon$ and magnetic energy $\varepsilon_{b}$. Since the large-scale magnetic energy dissipation is practically zero for every simulation performed, we ignore it from our analysis, as it plays no role. These measures are based on the dissipation rates from each of the three dissipation terms, and are defined in the following way:

$$
\varepsilon_{-} \equiv \nu_{-}\left\langle\left|\nabla^{-1} \boldsymbol{v}\right|^{2}\right\rangle / I, \quad \varepsilon \equiv \nu\left\langle\left|\nabla^{2} \boldsymbol{v}\right|^{2}\right\rangle / I, \quad \varepsilon_{b} \equiv \eta\left\langle\left|\nabla^{2} \boldsymbol{b}\right|^{2}\right\rangle / I
$$

Energy balance at steady state tells us that $\varepsilon_{-}+\varepsilon+\varepsilon_{b}=1$. In the limit of large Reynolds number and large forcing wavenumber, none of the energy injected is dissipated at the forcing scale and proper inertial ranges are formed. In this case, the dissipation rate at large scales represents the fraction of energy cascading to large scales, and similarly for the dissipation rate at small scales. Our runs do not reach these idealized limits. The lack of scale separation between the forcing and large-scale dissipation will manifest itself in two related ways in this paper: (i) the large scale dissipation rate will remain nonzero despite zero average inverse cascade, because some energy that is being injected at $k_{f}$ will be dissipated by the large-scale dissipation mechanism $\left(\varepsilon_{-} \leqslant 0.1\right.$ for the $3 \mathrm{D}$ runs), and (ii) when layers form in Regime III, both the 3D runs and 2D3C runs show an increase in large-scale dissipation rate, not because of the presence of an inverse cascade, but because the layers form coherent structures near the forcing scale, their energy grows and hence a stronger large-scale dissipation rate is achieved. These jumps in $\varepsilon_{-}$denote the presence of shear layers, as discussed in Section 4. To complement these estimates for energy cascades, we will look at the normalized spectral energy flux:

$$
\begin{aligned}
& \Pi_{K E}(k) \equiv\left\langle\boldsymbol{v}^{<k} \cdot(\boldsymbol{v} \cdot \nabla \boldsymbol{v})\right\rangle / I, \\
& \Pi_{M E}(k) \equiv-\left\langle\boldsymbol{v}^{<k} \cdot\left(\left(\boldsymbol{B}_{\mathbf{0}}+\boldsymbol{b}\right) \cdot \nabla \boldsymbol{b}\right)\right\rangle / I+\left\langle\boldsymbol{b}^{<k} \cdot\left(\boldsymbol{v} \cdot \nabla \boldsymbol{b}-\left(\boldsymbol{B}_{\mathbf{0}}+\boldsymbol{b}\right) \cdot \nabla \boldsymbol{v}\right)\right\rangle / I,
\end{aligned}
$$

where $\boldsymbol{v}^{<k}$ stands for a filtering of the velocity $\boldsymbol{v}$ in Fourier space so that only the wavenumbers with modulus smaller than $k$ are kept. The flux $\Pi(k)$ expresses the rate at which energy is flowing out of scales larger than $2 \pi / k$ due to nonlinear interactions, normalized by the energy injection rate. Therefore, if energy is going from large to small 

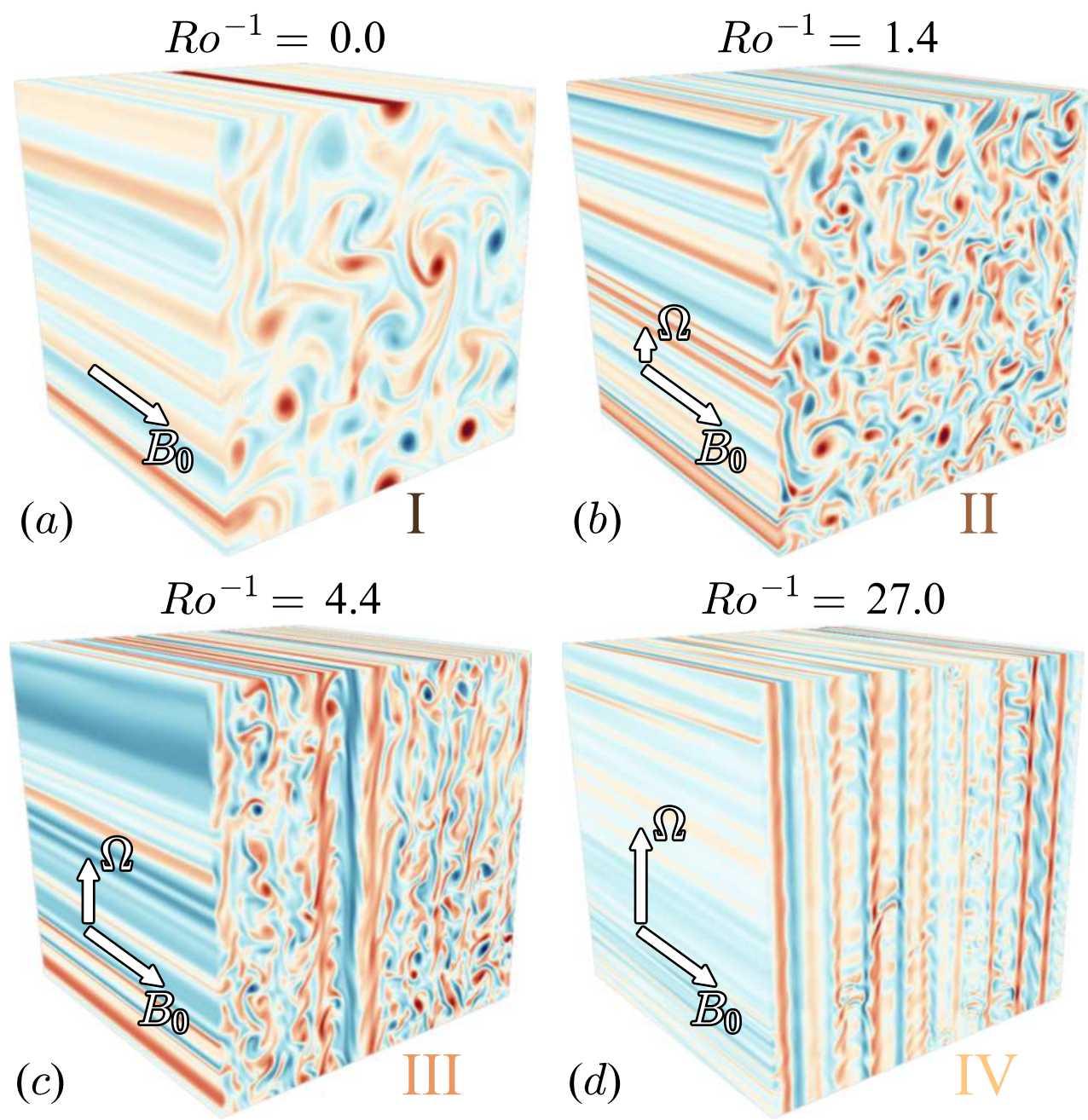

Figure 1. Snapshots of the field-aligned vorticity $\omega=\hat{x} \cdot(\nabla \times \boldsymbol{v})$, representing, from top left to bottom right, Regimes I $(a)$, II $(b)$, III $(c)$, and IV $(d)$, as rotation rate is increased. The red colors represent positive vorticity whereas the blue represents negative vorticity. Regime I is characterized by a bidirectional cascade, Regime II a purely forward cascade, Regime III the formation of strong shear layers (seen here in the middle of the domain), and Regime IV magnetically active shear layers. Regimes I-III have a negligible induced magnetic energy, unlike Regime IV whose magnetic energy dominates the dynamics (Figure 2).

scales, the energy flux will be positive, and vice versa. Finally, to quantify the amount and type of energy at each scale, we will also look at the energy spectra:

$$
E_{K E}(k) \equiv \frac{1}{2} \sum_{|\boldsymbol{k}|=k}|\widehat{\boldsymbol{v}}|^{2}(\boldsymbol{k}), \quad E_{M E}(k) \equiv \frac{1}{2} \sum_{|\boldsymbol{k}|=k}|\widehat{\boldsymbol{b}}|^{2}(\boldsymbol{k}),
$$

where $\widehat{\boldsymbol{v}}$ denotes the Fourier transform of $\boldsymbol{v}$.

Beginning from quasi-two-dimensional turbulence on the $y$ - $z$ plane at zero rotation, we find four distinct regimes as we increased rotation (Figure 1). Although not so apparent in the 3D simulations, these regimes are separated by seemingly sharp transitions, whose boundaries are determined in section 4 . 


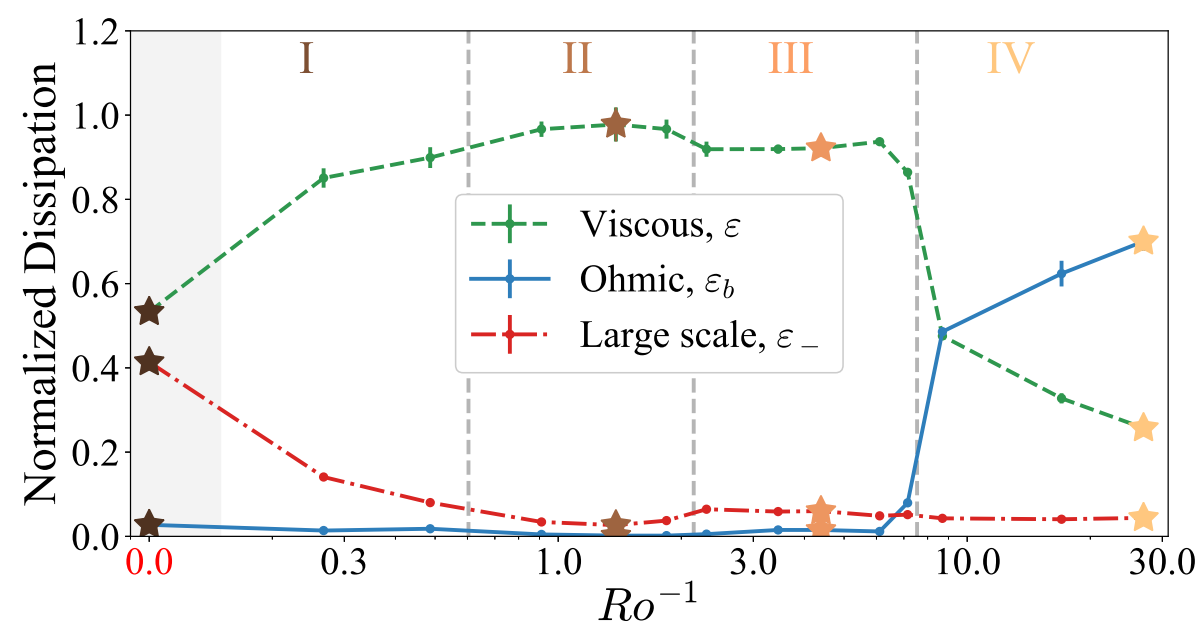

FIGURE 2. Dissipation rates normalized by the energy injection rate as a function of rotation rate measured by the inverse Rossby number $R o^{-1}$. The blue solid line shows the Ohmic dissipation rate due to the magnetic diffusion term, $\varepsilon_{b}$, the green dashed line shows the viscous dissipation rate, $\varepsilon$, and the red dash-dotted line shows the large-scale dissipation rate due to the hypoviscosity, $\varepsilon_{-}$. Each regime is labeled at the top, and the vertical dashed lines represent boundaries between regimes, chosen based on the two-dimensional runs in section 44 Stars represent runs whose snapshots are shown in Figure 1]

Regime I (Figure 1(a)), defined for runs with $R o^{-1}<0.6$, is characterized by the presence of a bidirectional cascade. This can be seen in Figure 2 as a non-zero large-scale dissipation rate as well as in Figure $3(e)$, where the spectral energy transfers show that about half of the energy injected by the forcing goes to large scales (negative $\Pi(k)$ ) and the other half goes to small scales (positive $\Pi(k)$ ). The fraction of energy that goes to larger scales decreases with increasing rotation (Figure 2). At zero rotation we don't have a purely inverse cascade $\left(\varepsilon_{-} \approx 1\right)$ due to a combination of finite background magnetic field strength and, as we will see in section 4 , the fact that we're forcing the out-of-plane velocity which acts as a passive scalar in the two-dimensionalized dynamics, thus contributing to a forward energy flux (Campagne et al. 2014; Biferale et al. 2017). Therefore, at zero rotation rate, the system is undergoing two independent cascades: an inverse energy cascade of horizontal kinetic energy and a forward cascade of the out-ofplane kinetic energy. If we were to force only the horizontal velocity components in the $k_{x}=0$ wavenumber plane, we would expect to see $\varepsilon_{-} \approx 1$ at zero rotation. Figure $3(a)$ shows the kinetic and magnetic energy spectra, which demonstrates that the magnetic energy is orders of magnitude smaller than the kinetic energy (particularly at large scales) and that the largest scales have the most energy, providing further confirmation of the presence of an inverse cascade. The spike of magnetic energy at the forcing scale is due to the excitation of Alfvén waves from the isotropic forcing. The eddy length scales seen in Figure 1 $(a)$ are set by a combination of the energy injection and the large-scale hypoviscosity coefficient.

Regime II (Figure 1 $(b)$ ), defined for runs with $0.6<R o^{-1}<2.1$, is characterized by a purely forward cascade of energy (Figures 2 and $3(f)$ ). This may come as a surprise, given that the dynamics are two-dimensional. The reason for this seemingly-contradictory state is that, while two-dimensional, all three velocity components are active in the dynamics and, furthermore, are coupled together with rotation. This results in a set of reduced two-dimensional, three-component (2D3C) equations which no longer conserve enstrophy, 

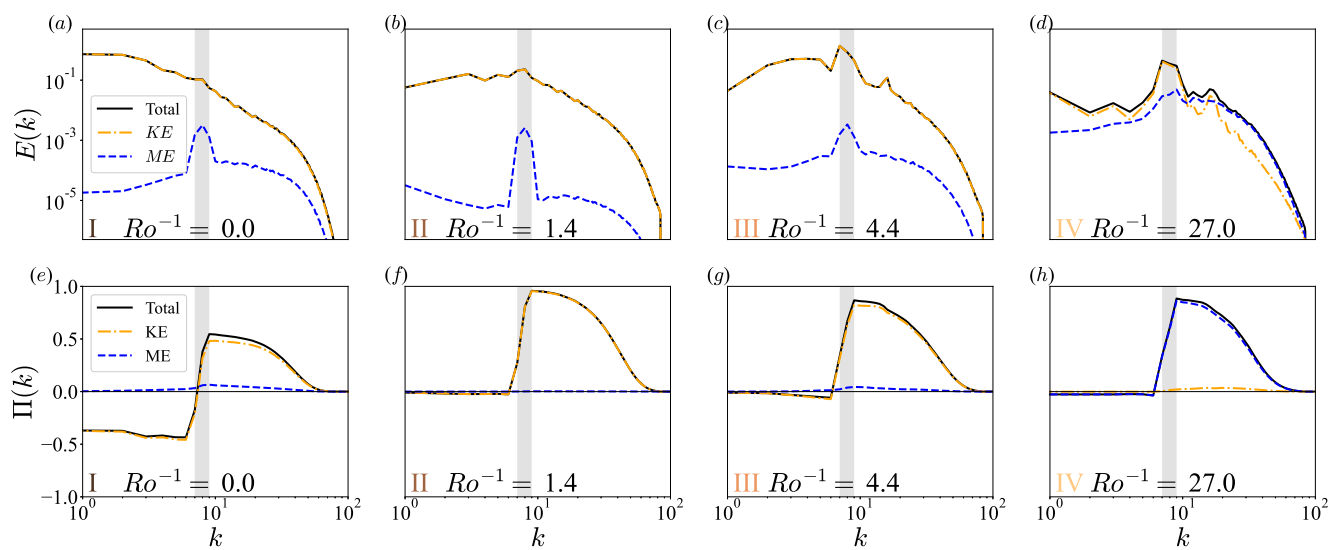

Figure 3. The time-averaged energy spectra (top row) and spectral energy flux (bottom row) for each Regime found in our simulations. The blue dashed line shows the magnetic components, either $E_{M E}$ or $\Pi_{M E}$, the orange dash-dotted line shows the kinetic components, $E_{K E}$ or $\Pi_{K E}$, and the solid black line shows their sum. The grey box represents the forcing range. These are from the same simulations shown in Figure 1 and which are starred in Figure 2.

making a forward cascade of energy possible. The rotating 2D3C system will be discussed and explored numerically in section 4 .

Regime III (Figure 1(c)), defined for runs with $2.1<R o^{-1}<7.5$, is characterized by the formation of strong shear-layers along the $y$-direction, consisting of uniform velocity in the $x-z$ plane. The shear layers form when the rotational constraint on the dynamics at large scales becomes sufficiently large, requiring that $\partial_{z} \boldsymbol{v} \approx 0$ at those scales. The combination of $\partial_{z}=\partial_{x}=0$ and incompressibility implies that $v_{y}=0$ (since we're in a periodic domain), and thus that the last remaining component of the nonlinear advection term $v_{y} \partial_{y}=0$ and nonlinearities are suppressed at large scales. Because of the suppressed nonlinearity at large scales, these shear layers form coherent structures that are fed directly by the forcing but that do not transfer that energy away, causing a build up of energy (not shown). The energy in the layers builds until a combination of the large-scale dissipation (Figure 2) and the nonlinear term (Figure 3 $(g)$ ) are able to remove energy from those scales. Regimes I-III have negligible induced magnetic energy, as is observed in simulations of MHD with a strong background field (Alexakis 2011; Sujovolsky \& Mininni 2016), and so the induced magnetic field plays an insignificant role in the dynamics. The magnetic fluctuations are also much smaller than $B_{0}-$ less than $0.5 \%$ of $B_{0}$ in Regimes I-III.

This changes, however, in Regime IV (Figure 1 $(d)$ ), defined for runs with $R o^{-1}>7.5$, where we have found the activation and growth of the induced magnetic field, which dominates both the energy as well as the nonlinear energy transfers (Figure 21). The nonlinear advection term in the momentum equation is suppressed for practically all scales (Figure 3(h)), leading to laminar-like shear-layer structures (Figure 1( $d)$ ) and a turbulent magnetic field which is responsible for the nonlinear transfers of energy across scales, via the Lorentz force and the magnetic induction equation. The shear layer spacing in Figure 1 $(d)$ is set by the forcing scale. In this regime, significant induced magnetic field fluctuations occur both parallel and perpendicular to the background magnetic field, with a magnitude of about $3 \%$ of $B_{0}$.

We expect the boundaries between Regimes I-III to be independent of $M^{-1}$, as they are part of the asymptotic 2D3C $\mathrm{HD}$, whose sole parameter is the rotation rate. We 


$\begin{array}{cccccccccc}\text { System } & B_{0} & M^{-1} & \Omega & k_{f} & \text { Forcing type } & \nu & \nu_{-} & \text {Resolution } & \text { Count } \\ (2.1)-(2.3) & 13.3 & 84 & {[0-16.6]} & 8-10 & \text { Constant Amplitude } & 6.6 \mathrm{e}-7 & 0.06 & 256^{3} & 14 \\ (2.1)-(2.3) & 6.6 & 44 & {[0-7.3]} & 8-10 & \text { Constant Amplitude } & 6.6 \mathrm{e}-7 & 0.06 & 256^{3} & 11 \\ (2.1)-(2.3) & 3.3 & 23 & {[0-7.3]} & 8-10 & \text { Constant Amplitude } & 6.6 \mathrm{e}-7 & 0.06 & 256^{3} & 5 \\ (4.1)-(4.2) & \infty & \infty & {[0-20]} & 12 & \text { Random } & 4 \mathrm{e}-7 & 1.0 & 512^{2} & 23\end{array}$

TABLE 1. A summary of the runs performed for this work. All runs have hyper- and hypo-viscosity of the same order (section 3). For runs with a magnetic field, $\mu=\nu$ and $\mu_{-}=\nu_{-}$. The simulations used Alfvénic units so that $B_{0} / \sqrt{\rho_{0} \mu_{0}} \rightarrow B_{0}$ and the other $\rho_{0}$ was absorbed into the pressure. The values are non-dimensionalized by $L$ and the forcing amplitude $f_{0}$ (or $I_{0}$ for random forcing), so that $k_{f}$ is the forcing mode number and the forcing amplitude (for constant amplitude forcing) or energy injection rate (for the random forcing) are both 1 in these units. The typical velocity, $U$, was calculated after-the-fact for each run using $U^{3} \equiv I k_{f}^{-1}$, where $I \equiv\langle\boldsymbol{f} \cdot \boldsymbol{v}\rangle$ is the time- and space-averaged energy injection rate. The count is the number of runs in that set.

confirm this in the next section, which deals specifically with this asymptotic set of equations, by showing that the regime transitions happen for the same values of $R o^{-1}$. The transition from Regime III to IV is of a different nature and represents a breakdown of the hydrodynamic behavior found for lower rotation rates. This transition is found to be $M^{-1}$-dependent, and will be discussed briefly in section 5

\section{Comparison to rotating two-dimensional, three-component model}

Regimes I-III can be better understood by considering the asymptotic limit of equations (2.1) - 2.3) when taking $M^{-1} \rightarrow \infty$ and keeping $R o^{-1} \sim \mathcal{O}(1)$ and the domain size fixed. The choice of keeping the domain size fixed is based on the fact that we are motivated mostly by astrophysical settings in confined geometries, in the presence of a strong background magnetic field. We acknowledge, however, that in many other astrophysical settings, such as the extended atmosphere of stars or the interstellar medium, a confined geometry may not be the best representative system to study. In such systems, a more appropriate limit might include taking the domain size to infinity at the same time as the $M^{-1}$, so as to prevent the exact two-dimensionalization of the flow (Thess \& Zikanov 2007; Gallet \& Doering 2015). The limiting equations in this case would resemble more the Reduced MHD system, derived for tokamaks but used also to study some astrophysical systems, in which the flow is highly anisotropic, yet still three-dimensional (Strauss 1976; Oughton et al. 2017).

Our limiting procedure, with fixed domain size, is similar to that done in Montgomery \& Turner (1981), with the exception that we include the Coriolis term, and so we only briefly discuss it here. Assuming a background magnetic field in the $x$ direction and a rotation axis along the $z$ direction, the process results in a set of three dynamical equations and one nonlinear constraint for the three variables: $\psi(y, z, t)$ the streamfunction for the in-plane velocities, $v_{x}(y, z, t)$ the out-of-plane velocity, and $A(y, z, t)$ the potential for the in-plane magnetic field. This novel constraint, which results from the presence of the Coriolis term, states that either $\delta A / \delta v_{x}=0$ or $v_{y}=\partial_{z} \psi=0$, where the former is the functional derivative of $A$ with respect to $v_{x}$. Our three-dimensional simulations from section 3 seem to be consistent with these constraints, where, in Regimes I-III for $R o^{-1}<7.5$, we have $A \approx 0$ but $v_{y} \neq 0$ and, in Regime IV for $R o^{-1}>7.5$, we have $A \neq 0$ but $v_{y}=\partial_{z} \psi \approx 0$. For the purposes 
of studying the reduced dynamics of Regimes I-III, we assume $A=0$, knowing that it would not capture the transition to Regime IV. The resulting equations form the two-dimensional, three-component (2D3C) system with in-plane rotation:

$$
\begin{gathered}
\frac{\partial v_{x}}{\partial t}+\left[v_{x}, \psi\right]=2 \Omega \frac{\partial \psi}{\partial z}+\nu \nabla_{\perp}^{2} v_{x}+f_{x}, \\
\frac{\partial \omega}{\partial t}+[\omega, \psi]=2 \Omega \frac{\partial v_{x}}{\partial z}+\nu \nabla_{\perp}^{2} \omega+f_{\omega},
\end{gathered}
$$

where $[F, G] \equiv \partial_{y} F \partial_{z} G-\partial_{y} G \partial_{z} F=0, \nabla_{\perp}=\left(0, \partial_{y}, \partial_{z}\right), \omega=\hat{x} \cdot(\nabla \times \boldsymbol{v})=-\nabla_{\perp}^{2} \psi$ is the out-of-plane vorticity (of the in-plane velocities), $\perp$ implies the directions perpendicular to the background magnetic field, and $f_{\omega}=\hat{x} \cdot\left(\nabla_{\perp} \times \boldsymbol{f}_{\perp}\right)$. One could equivalently arrive at (4.1) and (4.2) by taking rotating 3D HD and requiring that the velocity field doesn't depend on $x$. If considering an arbitrary angle between the background field and rotation, only the perpendicular projection of the rotation vector on the background field enters the model. For example, supposing without loss of generality that $\boldsymbol{B}_{\mathbf{0}}=B_{0} \hat{\boldsymbol{x}}$ and $\boldsymbol{\Omega}=\Omega(\sin (\theta) \hat{\boldsymbol{z}}+\cos (\theta) \hat{\boldsymbol{x}})$, then the Coriolis terms on the right-hand-side of (4.1) and (4.2) will be multiplied by $\sin (\theta)$. This asymptotic model is in the same spirit as some of the magnetized quasigeostrophic models used in astrophysical applications (Aurnou et al. 2015; Maffei et al. 2019), but it is important to note that here we have taken $M^{-1} \rightarrow \infty$ while keeping $R o^{-1} \sim \mathcal{O}(1)$, whereas the magnetized quasigeostrophic models take the rapidly rotating limit first. As is discussed in section 5 , these limits are not expected to be the same.

The Coriolis force now couples the two equations together, making what would otherwise be a passive tracer into an active one. In fact, for non-zero rotation, it can be shown that the $2 \mathrm{D} 3 \mathrm{C}$ rotating system conserves kinetic energy and helicity:

$$
\begin{aligned}
K E & =\frac{1}{2} \int v_{x}^{2}+|\nabla \psi|^{2} d^{2} x, \\
H & =\int v_{x} \omega d^{2} x .
\end{aligned}
$$

These are the same conserved quantities as in 3D HD, but we emphasize that the dynamics are two-dimensional and are occurring on the $y-z$ plane. This is in contrast to the case of zero rotation, where the system conserves (separately) the in-plane kinetic energy $\int|\nabla \psi|^{2} d^{2} x$ and the out-of-plane kinetic energy $\int v_{x}^{2} d^{2} x$, as well as the enstrophy, $\int \omega^{2} d^{2} x$. The conservation of enstrophy can be shown to prevent the existence of a forward cascade of in-plane kinetic energy (Fiortoft 1953; Kraichnan 1967; Alexakis \& Biferale 2018). Without the restriction of enstrophy conservation, though, the kinetic energy may go downscale in a forward cascade, even if one doesn't force the out-of-plane component.

We solve equations (4.1) and (4.2), with modified hyper- and hypo-viscosities as in the $3 \mathrm{D}$ simulations, numerically in a doubly-periodic domain of side length $2 \pi L$ using the $2 \mathrm{D}$ predecessor of GHOST. The code can be found in a public Github repository Benavides 2020). Unlike the 3D runs, whose forcing function had a constant amplitude in time, the 2D3C runs have random (white-in-time) forcing. At each time step, a wavenumber $\boldsymbol{k}_{r}$ of magnitude $k_{f}$ is chosen at random, and $\hat{f}_{\omega}(\boldsymbol{k})$ (Fourier transform of $f_{\omega}$ ) is set to zero everywhere except for at $\boldsymbol{k}_{r}$, where it had a magnitude $k_{f} \sqrt{2 I_{0} / \Delta t}$ (Chan et al. 2012). This has the effect of setting the energy injection rate for the in-plane flow to be $I=\left\langle\psi f_{\omega}\right\rangle=I_{0}$ on average, with $I_{0}$ being an input parameter of the simulation. The same forcing is applied for $f_{x}$, but with an amplitude of $\sqrt{2 I_{0} / \Delta t}$ instead, giving the same results. Therefore, half of the energy is injected into the in-plane flow and the other 

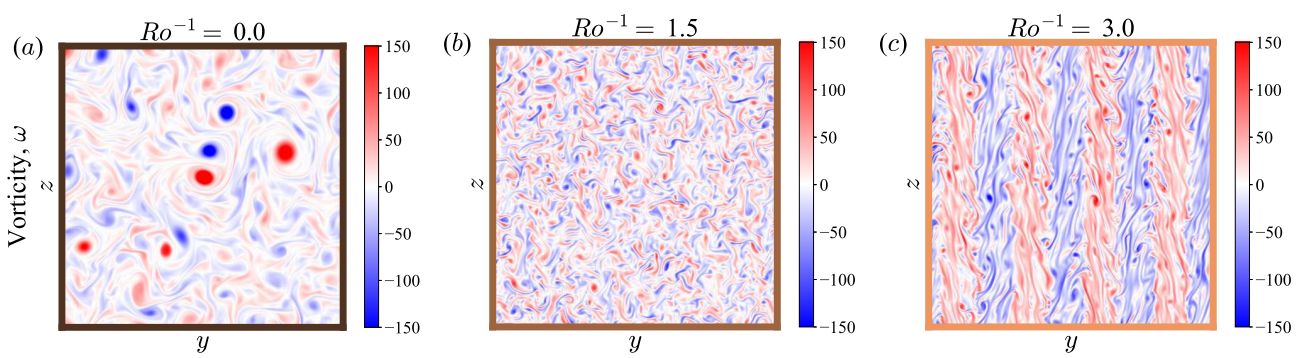

FiguRE 4. Snapshots of the out-of-plane vorticity, $\omega=\hat{x} \cdot(\nabla \times \boldsymbol{v})=-\nabla_{\perp}^{2} \psi$, for the 2D3C rotating simulations, representing, from left to right, Regimes I ( $a)$, II ( $b)$, and III $(c)$, as rotation is increased. We see striking similarities to Figure 1 confirming that the asymptotic 2D3C model captures the different regimes found in the 3D simulations of section 3.

half in the out-of-plane velocity. We nondimensionalize all dynamical variables as before, using $L$ and now the energy injection rate parameter $I_{0}$. For all of the runs reported $k_{f}=12$. See Table 1 for details on the runs.

The goal of these simulations is to reproduce the parameter sweep performed in section 3. but with the added advantage of working with a two-dimensional code, thus allowing a larger quantity of runs, higher resolutions (larger Reynolds numbers, around 600), and longer time integration. We have performed $23 \mathrm{runs}$, with $R_{O^{-1}}$ ranging from 0 to about 5 , at four times the horizontal resolution. Our results confirm the presence of Regimes IIII, going from a bidirectional cascade to a forward cascade to a shear-layer configuration (Figure 4).

At zero rotation we see a bidirectional cascade with half of the injected energy going to large scales and half going to small scales (Figure 5), similar to what was found in the $3 \mathrm{D}$ runs (Figure 2). For the 2D3C rotating system this is the case because of the choice of forcing, which injects half of the energy to the in-plane flow and the other half to the out-of-plane velocity. Since the two flows are completely decoupled at zero rotation, they each follow the standard behavior observed in 2D and passive tracer turbulence, that is, an inverse and forward cascade of energy, respectively. As we increase rotation, the Coriolis force couples the two fields, enstrophy is no longer conserved, and the inplane velocities no longer cascade all the injected energy to large scales, resulting in a bidirectional cascade with decreasing inverse energy flux. There is an approximately linear approach to zero inverse energy flux, and at $R o^{-1} \approx 0.6$ there is a transition to a purely forward cascade. With a larger number of simulations, Regimes I and II are much more clearly separated, and their transition appears to be sharp (Figure 5). This transition is qualitatively similar to other bidirectional to forward cascade transitions seen in other studies and could hint at a universal mechanism responsible for this transition (Seshasavanan et al. 2014; Seshasayanan \& Alexakis 2016a; Benavides \& Alexakis 2017; van Kan \& Alexakis 2020).

Upon further increase of the rotation, the forward cascade regime (Regime II) transitions to a shear layer configuration (Figure $4(c)$ ), entering Regime III. This corresponds to the case when the Coriolis force dominates at large scales, making the dominant balance in equations (4.1) and (4.2) $\partial_{z} \psi \approx \partial_{z} v_{x} \approx 0$, hence the layers. There are a few differences in the morphology of the shear layers seen for these runs, compared to Regime III in the 3D simulations (Figure 1 $(c)$ ). Here they take up the whole domain and also appear to equilibrate at scales larger than the forcing, through a series of mergers (not shown). Neither of these characteristics are seen in the shear layers of the $3 \mathrm{D}$ simulations. We believe this is due to a few factors, including the longer integration 


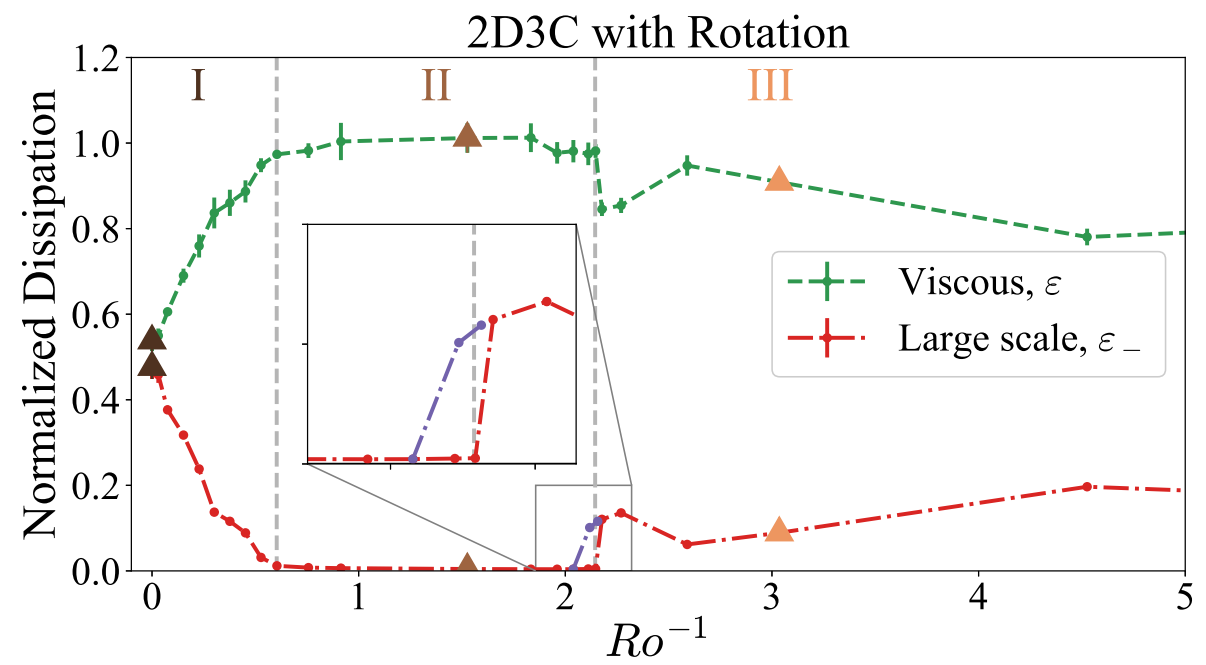

FiguRE 5. Dissipation rates normalized by the energy injection rate as a function of rotation rate measured by the inverse Rossby number $R o^{-1}$. The green dashed line shows the viscous dissipation rate, $\varepsilon$, the red dash-dotted line shows the large-scale dissipation rate due to the hypoviscosity, $\varepsilon_{-}$, and the purple dash-dotted line shows the same but for hysteresis runs initialized with layers. Each regime is labeled at the top, and the vertical dashed lines represent boundaries between regimes. These denoted boundaries are placed at the same value of $R o^{-1}$ as those seen in Figure 2 Triangles represent runs whose snapshots are shown in Figure 4.

times and the change in forcing. A surprising feature of this transition, revealed by the better-resolved parameter sweep, is that it is discontinuous (Figure 5) 6. Discontinuities are a characteristic of subcritical bifurcations, which should also display hysteresis. By initializing in the layered regime, we confirmed the presence of hysteresis as we reduced the rotation rate (Figure 5 inset).

Despite differences in the forcing, Reynolds numbers, and values of $M^{-1}$, the regime transitions seem to occur for the same values of $R o^{-1}$, suggesting that the rotating 2D3C system successfully describes the dynamics observed in the 3D simulations from section 3 and that Regimes I-III are robust properties of the system. The two-dimensional asymptotic system has allowed us to perform a more detailed parameter sweep of this parameter space, and has revealed sharp transitions and nontrivial behavior near those transitions which we did not anticipate from the 3D simulations.

\section{Discussion \& Conclusions}

We have investigated the turbulent dynamics of rotating magnetohydrodynamics in the presence of a strong uniform background magnetic field perpendicular to the rotation axis. Our investigations have revealed surprising behavior, confirmed both by threedimensional and a two-dimensional three-component asymptotic model, as rotation rate

$\dagger$ An increase in large-scale dissipation marks this transition not because an inverse cascade forms (a weakness of this measure), but because of a lack of separation of scales. The layers form at or near the forcing scale and remain there as coherent structures, fed directly by the forcing, resulting in a build up of energy at those scales. This, in turn, results in a larger dissipation rate from the large-scale dissipation. If we were to perform runs at a larger $k_{f}$, this effect would disappear. The discontinuous transition is also observed in the kinetic energy, which is not shown. 
is increased. We observed the weakening of the inverse cascade, a transition to a purely forward cascade for relatively weak rotation, and eventually a shear-layer regime at larger rotation rates. These results were obtained in a specific situation: orthogonal rotation and guide field at unit magnetic Prandtl number. However, the derivation of the asymptotic 2D3C model allows us to generalise Regimes I-III to the more realistic situation of an arbitrary angle between rotation and guide field at low magnetic Reynolds number. First, for an arbitrary angle $\theta$ between rotation vector and guide field, the reduced model is given by equations (4.1) and (4.2) where $2 \Omega$ is replaced by $2 \Omega \sin (\theta)$, the consequence being that the results in Figure 5 carry over with $R o^{-1}$ replaced by $R o^{-1} \sin (\theta)$ in the $x$-axis. Second, the 2D3C model illustrates the asymptotic limit in which the guide field is so strong that it prevents any $x$-dependence. The same phenomenon arises for the low magnetic Reynolds numbers that characterize transitions regions in planetary interiors, see Gallet \& Doering (2015) for a rigorous proof in an idealized setting. The consequence is that we expect the very same reduced 2D3C model to hold at low magnetic Reynolds number, starting either from the full MHD equations or from their low-magnetic-Reynolds-number quasi-static approximation. We thus conclude that Regimes I-III carry over to the planetary relevant situation of an arbitrary angle between rotation and guide field, together with a low magnetic Reynolds number (by contrast, the magnetically active Regime IV will be affected by changes in magnetic Prandtl number).

We should also stress the fact that our study focuses on finite-size domains: motivated by transitional layers in planetary interiors, we have restricted attention to a numerical domain that is finite both along the direction of the rotation vector and the local direction of the large-scale magnetic field. By contrast, an idealized turbulent cloud allowed to develop arbitrarily large structures would never achieve exact two-dimensionalization (Davidson 2013; van Kan \& Alexakis 2020, 2021), and it's possible that in those cases the Reduced MHD description might be more relevant (Strauss 1976; Oughton et al. 2017).

The strong sensitivity of the inverse cascade to in-plane rotation could have significant implications for the morphology of astrophysical flows, which often have both rotation and a background magnetic field. Even for relatively weak rotation $\left(R o^{-1} \sim 1\right)$ the inverse cascade is entirely suppressed. Seeing as an inverse cascade is considered to be necessary for the formation of jets on gas giant planets, this phenomenon could be a tentative alternative explanation for the weakening of the jets in the depths of their atmospheres, as seen by the Juno mission on Jupiter (Kaspi et al. 2018). In the outer electricallyneutral regions the jets can form because of the rapid rotation. These rotation-aligned jets may penetrate deep into the interior until they reach the low $R e_{m}$ ionized regions of the atmosphere whose turbulent dynamics suppress the jets via Ohmic dissipation (Liu et al. 2008). Our work reveals another potential alternative, where a misalignment of the rotation and background field cause the localized turbulent dynamics to cascade energy forward instead of inversely, thereby taking away the dynamical origin of the jets. Apart from the astrophysical implications, the rotating 2D3C model might be of interest to those studying phase transitions in turbulence (Alexakis \& Biferale 2018) particularly those interested in the transition from a forward to a bidirectional cascade, since, as far as we are aware, this model is the only two-dimensional hydrodynamical one with this behavior.

At the largest rotation rotates, our 3D simulations showed a sudden activation of the induced magnetic field, signaling the breakdown of the purely hydrodynamic asymptotic model. The velocity field remained 2D3C, but the dynamics differed significantly from the hydrodynamic shear-layer regime and were dominated by an induced three-dimensional 


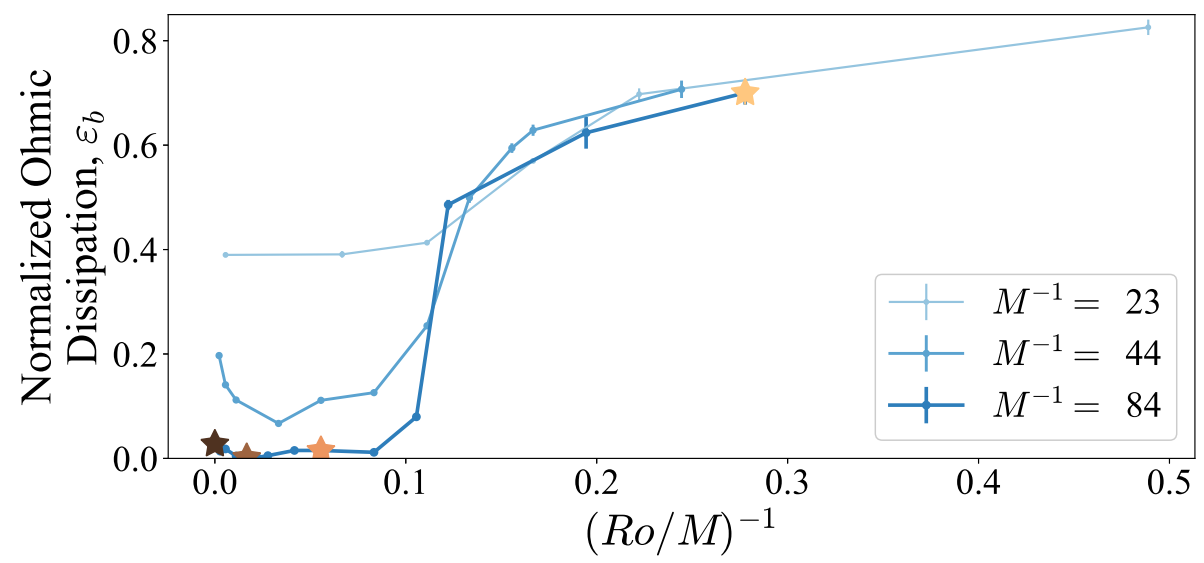

Figure 6. Normalized Ohmic dissipation, $\varepsilon_{b}$, versus $(R o / M)^{-1}$. The Ohmic dissipation represents a measure of how active the induced magnetic field is in the dynamics. We see that, for three values of $M^{-1}$, the induced magnetic field begins to dominate the dynamics once $(R o / M)^{-1}>0.1$, in other words when $R o^{-1}>0.1 M^{-1} .(R o / M)^{-1}$ is also referred to as the Lehnert number (Lehnert 1955). Stars represent runs whose snapshots are shown in Figure 1

magnetic field. Although the 2D3C model breaks down, given the three-dimensionality of the magnetic field, it's possible this transition could be studied with the Reduced MHD system (Strauss 1976; Oughton et al. 2017). A series of simulations at lower $M^{-1}$ values (Table 1) reveal that the transition happens when $M \sim R o$, which represents roughly the point at which the inertial wave frequency begins to dominate over the Alfvén wave frequency (Figure 6] Appendix A). Interestingly, this transition sharpens towards a critical value as the background magnetic field strength increases. Therefore, when considering the limit of strong rotation and strong background magnetic field, the order in which those limits are taken matters. If $R o^{-1}<0.1 M^{-1}$, then one would expect a hydrodynamical regime, whereas if $R o^{-1}>0.1 M^{-1}$ a magnetically-dominated regime is expected.

\section{Acknowledgments}

The authors thank the referees for helpful feedback which has improved the quality and clarity of the manuscript.

\section{Funding}

This research was carried out in part during the 2019 Summer School at the Center for Computational Astrophysics, Flatiron Institute. The Flatiron Institute is supported by the Simons Foundation. SJB acknowledges funding from a grant from the National Science Foundation (OCE-1459702) and from the National Aeronautics and Space Administration (Award Number: 80NSSC20K1367) issued through the Future Investigators in NASA Earth and Space Science and Technology (NNH19ZDA001N-FINESST) within the NASA Research Announcement (NRA): Research Opportunities in Space and Earth Sciences (ROSES-2019).

\section{Declaration of Interests}

The authors report no conflict of interest. 

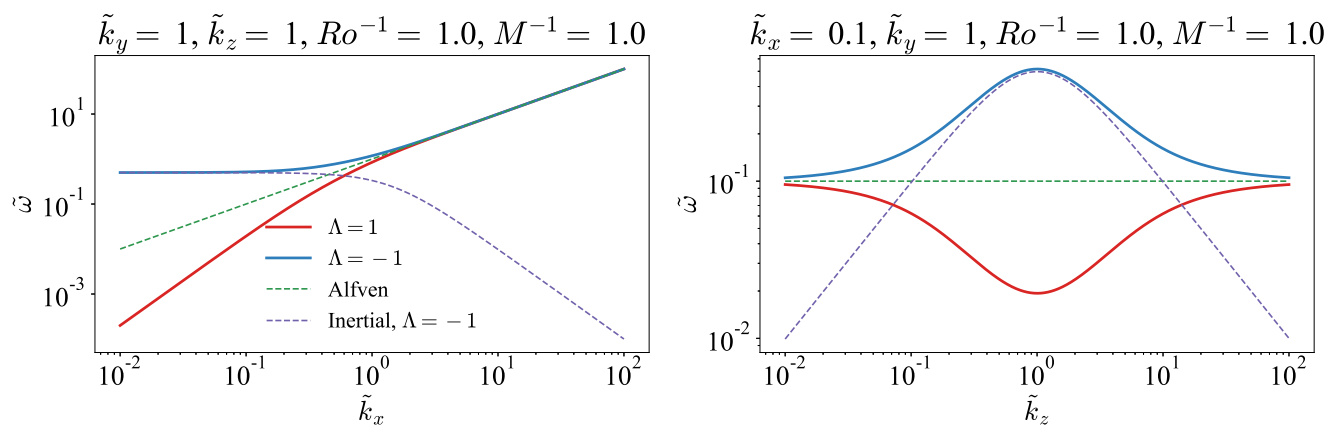

Figure 7 . The dispersion relation for waves in the $B \Omega$-MHD system, in the case where $\boldsymbol{B}_{\mathbf{0}}=B_{0} \hat{\boldsymbol{x}}$ and $\boldsymbol{\Omega}=\Omega \hat{\boldsymbol{z}}$ (equation (A3)). The green dashed line shows the Alfvén wave dispersion relation, and the purple dashed line shows that of an inertial wave. The low frequency branch with $\Lambda=1$ is similar to the magnetostrophic mode in the case when $\boldsymbol{B}_{\mathbf{0}}$ and $\boldsymbol{\Omega}$ are aligned (Galtier 2014).

\section{Data availability statement}

The three-dimensional $B \Omega$-MHD runs were done using Benavides (2019). The twodimensional 2D3C runs were done using Benavides (2020). The time-averaged data, which includes run parameters, and a script for creating the figures can be found at https://doi.org/10.6084/m9.figshare.16888135.v1. The time-series used to create this data can be provided upon request to the corresponding author.

\section{Appendix A. Wave Dispersion Relation}

In the inviscid, perfectly-conducting, and force-free case, the linearized $B \Omega$-MHD system admits wave solutions. Taking $\boldsymbol{v}$ of the form $\boldsymbol{v}=\hat{\boldsymbol{v}} \exp i(\boldsymbol{k} \cdot \boldsymbol{x}-\omega t)$ and plugging this into the linearized versions of equations (2.1)-(2.3), after some algebra we end up with:

$$
\omega^{2}(\boldsymbol{k} \times \hat{\boldsymbol{v}})=2 i \omega(\boldsymbol{k} \cdot \boldsymbol{\Omega}) \hat{\boldsymbol{v}}+\left(\boldsymbol{k} \cdot \boldsymbol{B}_{\mathbf{0}}\right)^{2} \boldsymbol{k} \times \hat{\boldsymbol{v}} .
$$

Next we introduce the helical orthonormal basis for $\hat{\boldsymbol{v}}, \hat{\boldsymbol{v}}(\boldsymbol{k})=v_{k}^{+} \hat{\boldsymbol{h}}_{k}^{+}+v_{k}^{-} \hat{\boldsymbol{h}}_{k}^{-}$, where $\boldsymbol{k} \times \hat{\boldsymbol{h}}_{k}^{\Lambda}=-i \Lambda|\boldsymbol{k}| \hat{\boldsymbol{h}}_{k}^{\Lambda}$ and $\Lambda= \pm 1$ indicates the sign of the helicity of $\hat{\boldsymbol{h}}_{k}^{\Lambda}$ (Herring 1974; Alexakis 2017). Introducing these basis and dotting equation (A 1) with $\hat{\boldsymbol{h}}_{k}^{\Lambda}$ we arrive at the dispersion relation. We normalize the frequency with the eddy turnover frequency, $k_{f} U$, and the wavevector $\boldsymbol{k}$ with $k_{f}$, resulting in our final expression for the dispersion relation:

$$
\tilde{\omega}(\tilde{\boldsymbol{k}} ; \Lambda)=-\frac{1}{2} \frac{\tilde{\boldsymbol{k}} \cdot \hat{\boldsymbol{x}}_{\|}^{\Omega}}{\Lambda \tilde{k} R o} \pm \frac{1}{2} \sqrt{\left(\frac{\tilde{\boldsymbol{k}} \cdot \hat{\boldsymbol{x}}_{\|}^{\Omega}}{\tilde{k} R o}\right)^{2}+4\left(\frac{\tilde{\boldsymbol{k}} \cdot \hat{\boldsymbol{x}}_{\|}^{B_{0}}}{M}\right)^{2}},
$$

where $\tilde{k} \equiv\left(\tilde{k}_{x}^{2}+\tilde{k}_{y}^{2}+\tilde{k}_{z}^{2}\right)^{1 / 2}, \tilde{k}_{i} \equiv k_{i} / k_{f}$, and $\tilde{\omega} \equiv \omega /\left(k_{f} U\right)$. In the specific case of our study, where $\boldsymbol{B}_{\mathbf{0}}=B_{0} \hat{\boldsymbol{x}}$ and $\boldsymbol{\Omega}=\Omega \hat{\boldsymbol{z}}$, this simplifies to:

$$
\tilde{\omega}(\tilde{\boldsymbol{k}} ; \Lambda)=-\frac{1}{2} \frac{\tilde{k}_{z}}{\Lambda \tilde{k} R o} \pm \frac{1}{2} \sqrt{\left(\frac{\tilde{k}_{z}}{\tilde{k} R o}\right)^{2}+4\left(\frac{\tilde{k}_{x}}{M}\right)^{2}} .
$$

See Figure 7 for a visualization of this dispersion relation, which depends on $\tilde{k}_{x}, \tilde{k}_{z}$, and $\tilde{k}$. 


\section{REFERENCES}

Agrawal, R., Alexakis, A., Brachet, M. E. \& Tuckerman, L. S. 2020 Turbulent cascade, bottleneck, and thermalized spectrum in hyperviscous flows. Phys. Rev. Fluids 5, 024601.

Alexakis, A. 2011 Two-dimensional behavior of three-dimensional magnetohydrodynamic flow with a strong guiding field. Phys. Rev. E 84, 056330.

Alexakis, A. 2017 Helically decomposed turbulence. Journal of Fluid Mechanics 812, 752-770.

Alexakis, A. \& Biferale, L. 2018 Cascades and transitions in turbulent flows. Physics Reports 767-769, 1 - 101.

Arbic, B. K. \& Flierl, G. R. 2004 Effects of mean flow direction on energy, isotropy, and coherence of baroclinically unstable beta-plane geostrophic turbulence. Journal of Physical Oceanography 34 (1), $77-93$.

Armitage, P. J. 2011 Dynamics of protoplanetary disks. Annual Review of Astronomy and Astrophysics 49 (1), 195-236.

Aurnou, J.M., Calkins, M.A., Cheng, J.S., Julien, K., King, E.M., Nieves, D., Soderlund, K.M. \& Stellmach, S. 2015 Rotating convective turbulence in earth and planetary cores. Physics of the Earth and Planetary Interiors 246, 52-71.

Baker, N. T., Pothérat, A., Davoust, L. \& Debray, F. 2018 Inverse and direct energy cascades in three-dimensional magnetohydrodynamic turbulence at low magnetic reynolds number. Phys. Rev. Lett. 120, 224502.

Baklouti, F. S., Khlifi, A., Salhi, A., Godeferd, F., Cambon, C. \& Lehner, T. 2019 Kinetic-magnetic energy exchanges in rotating magnetohydrodynamic turbulence. Journal of Turbulence 20 (4), 263-284.

Bell, N. K. \& NazAREnko, S. V. 2019 Rotating magnetohydrodynamic turbulence. Journal of Physics A: Mathematical and Theoretical 52 (44), 445501.

Benavides, S. J. 2019 Geophysical high-order suite for turbulence. GitHub repository https://github.com/s-benavides/GHOST/tree/pre-release-2, Commit: 2caaa43aad8b9378ec154bfbc26e4c85d947a4cc.

Benavides, S. J. 2020 Rot2d3c. GitHub repository https://github.com/sbenavides/Rot2D3C, Commit: 2d511888b78c451fc7b26352916a6ff40ee98b93.

Benavides, S. J. \& Alexakis, A. 2017 Critical transitions in thin layer turbulence. Journal of Fluid Mechanics 822, 364-385.

Benavides, S. J. \& Flierl, G. R. 2020 Two-dimensional partially ionized magnetohydrodynamic turbulence. Journal of Fluid Mechanics 900, A28.

Biferale, L., Buzzicotti, M. \& Linkmann, M. 2017 From two-dimensional to threedimensional turbulence through two-dimensional three-component flows. Physics of Fluids 29 (11), 111101.

Bigot, B. \& Galtier, S. 2011 Two-dimensional state in driven magnetohydrodynamic turbulence. Phys. Rev. E 83, 026405.

Boffetta, G. \& Ecke, R. E. 2012 Two-dimensional turbulence. Annual Review of Fluid Mechanics 44 (1), 427-451.

Buzzicotti, M., Aluie, H., Biferale, L. \& Linkmann, M. 2018 Energy transfer in turbulence under rotation. Phys. Rev. Fluids 3, 034802.

Campagne, A., Gallet, B., Moisy, F. \& Cortet, P.-P. 2014 Direct and inverse energy cascades in a forced rotating turbulence experiment. Physics of Fluids 26 (12), 125112.

Celani, A., Musacchio, S. \& Vincenzi, D. 2010 Turbulence in more than two and less than three dimensions. Phys. Rev. Lett. 104, 184506.

Chan, C.-K., Mitra, D. \& Brandenburg, A. 2012 Dynamics of saturated energy condensation in two-dimensional turbulence. Phys. Rev. E 85, 036315.

Charney, J. G. 1971 Geostrophic turbulence. Journal of Atmospheric Sciences 28 (6), 1087 1095.

Chertkov, M., Connaughton, C., Kolokolov, I. \& Lebedev, V. 2007 Dynamics of energy condensation in two-dimensional turbulence. Phys. Rev. Lett. 99, 084501.

Cho, J. Y-K. \& Polvani, L. M. 1996a The emergence of jets and vortices in freely evolving, shallow-water turbulence on a sphere. Physics of Fluids 8 (6), 1531-1552.

Сно, J. Y-K. 2008 Atmospheric dynamics of tidally synchronized extrasolar planets. Philosophical Transactions of the Royal Society A: Mathematical, Physical and Engineering Sciences 366 (1884), 4477-4488. 
Cho, J. Y-K. \& Polvani, L. M. $1996 b$ The morphogenesis of bands and zonal winds in the atmospheres on the giant outer planets. Science 273 (5273), 335-337.

DAvidson, P. A. 2013 Turbulence in rotating, stratified and electrically conducting fluids. Cambridge University Press.

Deusebio, E., Boffetta, G., Lindborg, E. \& Musacchio, S. 2014 Dimensional transition in rotating turbulence. Phys. Rev. E 90, 023005.

Diamond, P. H., Iтон, S.-I., Iтон, K. \& Hahm, T. S. 2005 Zonal flows in plasma-a review. Plasma Physics and Controlled Fusion 47 (5), R35-R161.

Dietrich, W \& Jones, C A 2018 Anelastic spherical dynamos with radially variable electrical conductivity. Icarus 305, 15-32.

Favier, B., Godeferd, F. S., Cambon, C. \& Delache, A. 2010 On the twodimensionalization of quasistatic magnetohydrodynamic turbulence. Physics of Fluids $22(7), 075104$.

Favier, B. F.N., Godeferd, F. S. \& Cambon, C. 2012 On the effect of rotation on magnetohydrodynamic turbulence at high magnetic Reynolds number. Geophysical and Astrophysical Fluid Dynamics 106 (1), 89-111, arXiv: 1103.6236.

Fjortoft, R. 1953 On the changes in the spectral distribution of kinetic energy for twodimensional, nondivergent flow. Tellus 5 (3), 225-230.

French, M., Becker, A., Lorenzen, W., Nettelmann, N., Bethkenhagen, M., Wicht, J. \& RedmeR, R. 2012 Ab initio simulations for material properties along the jupiter adiabat. The Astrophysical Journal Supplement Series 202 (1), 5.

Frisch, U. 1995 Turbulence: the legacy of AN Kolmogorov. Cambridge University Press.

Fromang, S. 2005 The effect of mhd turbulence on massive protoplanetary disk fragmentation. Astronomy \& Astrophysics 441 (1), 1-8.

GALLET, B. 2015 Exact two-dimensionalization of rapidly rotating large-reynolds-number flows. Journal of Fluid Mechanics 783, 412-447.

Gallet, B. \& Doering, C. R. 2015 Exact two-dimensionalization of low-magnetic-reynoldsnumber flows subject to a strong magnetic field. Journal of Fluid Mechanics 773, 154-177.

Gallet, B. \& Ferrari, R. 2021 A quantitative scaling theory for meridional heat transport in planetary atmospheres and oceans. AGU Advances 2 (3).

Gallet, B. \& Young, W. R. 2013 A two-dimensional vortex condensate at high reynolds number. Journal of Fluid Mechanics 715, 359-388.

GaltieR, S. 2014 Weak turbulence theory for rotating magnetohydrodynamics and planetary flows. Journal of Fluid Mechanics 757 (6), 114-154.

Held, I. M. \& LARICheV, V. D. 1996 A scaling theory for horizontally homogeneous, baroclinically unstable flow on a beta plane. Journal of Atmospheric Sciences 53 (7), $946-952$.

HeRring, J. R. 1974 Approach of axisymmetric turbulence to isotropy. The Physics of Fluids $17(5), 859-872$.

Joos, M., Hennebelle, P. \& Ciardi, A. 2012 Protostellar disk formation and transport of angular momentum during magnetized core collapse. Astronomy \& Astrophysics 543, A128.

VAn Kan, A. \& Alexakis, A. 2019 Condensates in thin-layer turbulence. Journal of Fluid Mechanics 864, 490-518.

VAN KAN, A. \& AleXAKIS, A. 2020 Critical transition in fast-rotating turbulence within highly elongated domains. Journal of Fluid Mechanics 899, A33.

VAN KAN, A. \& AlEXAKIS, A. 2021 Energy cascades in rapidly rotating and stratified turbulence within elongated domains, arXiv: 2106.06973.

Kaspi, Y., Galanti, E., Hubbard, W. B., Stevenson, D. J., Bolton, S. J., Iess, L., Guillot, T., Bloxham, J., Connerney, J. E. P., CaO, H., Durante, D., Folkner, W. M., Helled, R., Ingersoll, A. P., Levin, S. M., Lunine, J. I., Miguel, Y., Militzer, B., PArisi, M. \& Wahl, S. M. 2018 Jupiter's atmospheric jet streams extend thousands of kilometres deep. Nature 555, 223.

Kraichnan, R. H. 1967 Inertial ranges in two-dimensional turbulence. The Physics of Fluids $10(7), 1417-1423$.

LEhNeRT, B. 1955 The decay of magneto-turbulence in the presence of a magnetic field and coriolis force. Quarterly of Applied Mathematics 12 (4), 321-341. 
Liu, J., Goldreich, P. \& Stevenson, D. J. 2008 Constraints on deep-seated zonal winds inside Jupiter and Saturn. Icarus 196 (2), 653-664.

Maffei, S., Calkins, M. A., Julien, K. \& Marti, P. 2019 Magnetic quenching of the inverse cascade in rapidly rotating convective turbulence. Phys. Rev. Fluids 4, 041801.

Marino, R., Pouquet, A. \& Rosenberg, D. 2015 Resolving the paradox of oceanic large-scale balance and small-scale mixing. Phys. Rev. Lett. 114, 114504.

Menu, M. D., Galtier, S. \& Petitdemange, L. 2019 Inverse cascade of hybrid helicity in b $\Omega$-mhd turbulence. Phys. Rev. Fluids 4, 073701.

Mininni, P. D. \& Pouquet, A. 2010 Rotating helical turbulence. i. global evolution and spectral behavior. Physics of Fluids 22 (3), 035105.

Mininni, P. D., Rosenberg, D., Reddy, R. \& Pouquet, A. 2011 A hybrid mpi-openmp scheme for scalable parallel pseudospectral computations for fluid turbulence. Parallel Computing 37 (6), 316 - 326.

Montgomery, D. \& Turner, L. 1981 Anisotropic magnetohydrodynamic turbulence in a strong external magnetic field. Physics of Fluids 24 (5), 825-831.

Moore, K. M., Yadav, R. K., Kulowski, L., CaO, H., Bloxham, J., Connerney, J. E.P., Kotsiaros, S., Jørgensen, J. L., Merayo, J. M.G., Stevenson, D. J., Bolton, S. J. \& Levin, S. M. 2018 A complex dynamo inferred from the hemispheric dichotomy of Jupiter's magnetic field. Nature 561 (7721), 76-78.

Nazarenko, S. 2007 2d enslaving of MHD turbulence. New Journal of Physics 9 (8), 307-307.

Otani, N. F. 1993 A fast kinematic dynamo in two-dimensional time-dependent flows. Journal of Fluid Mechanics 253, 327-340.

Oughton, S., Matthaeus, W. H. \& Dmitruk, P. 2017 Reduced MHD in astrophysical applications: Two-dimensional or three-dimensional? The Astrophysical Journal 839 (1), 2 .

Pouquet, A. \& Marino, R. 2013 Geophysical turbulence and the duality of the energy flow across scales. Phys. Rev. Lett. 111, 234501.

Pouquet, A., Rosenberg, D., Stawarz, J.E. \& Marino, R. 2019 Helicity dynamics, inverse, and bidirectional cascades in fluid and magnetohydrodynamic turbulence: A brief review. Earth and Space Science 6 (3), 351-369.

Rhines, P. B. 1975 Waves and turbulence on a beta-plane. Journal of Fluid Mechanics 69 (3), 417-443.

Seshasayanan, K. \& Alexakis, A. $2016 a$ Critical behavior in the inverse to forward energy transition in two-dimensional magnetohydrodynamic flow. Physical Review E 93 (1), 113.

Seshasayanan, K. \& Alexakis, A. $2016 b$ Turbulent 2.5-dimensional dynamos. Journal of Fluid Mechanics 799, 246-264.

Seshasayanan, K. \& Alexakis, A. 2018 Condensates in rotating turbulent flows. Journal of Fluid Mechanics 841, 434-462.

Seshasayanan, K., Benavides, S. J. \& Alexakis, A. 2014 On the edge of an inverse cascade. Physical Review E - Statistical, Nonlinear, and Soft Matter Physics 90 (5), 1-5.

Seshasayanan, K., Gallet, B. \& Alexakis, A. 2017 Transition to turbulent dynamo saturation. Phys. Rev. Lett. 119, 204503.

Shebalin, J. V. 2006 Ideal homogeneous magnetohydrodynamic turbulence in the presence of rotation and a mean magnetic field. Journal of Plasma Physics 72 (4), 507-524.

Simon, J. B., Bai, X.-N., Armitage, P. J., Stone, J. M. \& Beckwith, K. 2013 Turbulence in the outer regions of protoplanetary disks. ii. strong accretion driven by a vertical magnetic field. The Astrophysical Journal $\mathbf{7 7 5}$ (1), 73.

Simon, J. B., Bai, X.-N., Flaherty, K. M. \& Hughes, A. M. 2018 Origin of weak turbulence in the outer regions of protoplanetary disks. The Astrophysical Journal 865 (1), 10.

Smith, L. M., Chasnov, J. R. \& Waleffe, F. 1996 Crossover from two- to three-dimensional turbulence. Phys. Rev. Lett. 77, 2467-2470.

Smith, L. M. \& Waleffe, F. 1999 Transfer of energy to two-dimensional large scales in forced, rotating three-dimensional turbulence. Physics of Fluids 11 (6), 1608-1622.

Sмith, S. G. L. \& Tobias, S. M. 2004 Vortex dynamos. Journal of Fluid Mechanics 498, $1-21$. 
Sommeria, J. \& Moreau, R. 1982 Why, how, and when, mhd turbulence becomes twodimensional. Journal of Fluid Mechanics 118, 507-518.

Strauss, H. R. 1976 Nonlinear, three-dimensional magnetohydrodynamics of noncircular tokamaks. The Physics of Fluids 19 (1), 134-140.

Sujovolsky, N. E. \& Mininni, P. D. 2016 Tridimensional to bidimensional transition in magnetohydrodynamic turbulence with a guide field and kinetic helicity injection. Phys. Rev. Fluids 1, 054407.

Thess, A. \& Zikanov, O. 2007 Transition from two-dimensional to three-dimensional magnetohydrodynamic turbulence. Journal of Fluid Mechanics 579, 383-412.

Tobias, S.M. 2021 The turbulent dynamo. Journal of Fluid Mechanics 912, P1.

Tobias, S. M., Diamond, P. H. \& Hughes, D. W. $2007 \beta$-plane magnetohydrodynamic turbulence in the Solar Tachocline. The Astrophysical Journal 667, 113-116.

Vallis, G. K. 2017 Atmospheric and oceanic fluid dynamics. Cambridge University Press.

Vorobev, A., Zikanov, O., Davidson, P. A. \& Knaepen, B. 2005 Anisotropy of magnetohydrodynamic turbulence at low magnetic reynolds number. Physics of Fluids 17 (12), 125105.

Xia, H., Byrne, D., Falkovich, G. \& Shats, M. 2011 Upscale energy transfer in thick turbulent fluid layers. Nature Physics 7 (4), 321-324.

Xia, H., Punzmann, H., Falkovich, G. \& Shats, M. G. 2008 Turbulence-condensate interaction in two dimensions. Phys. Rev. Lett. 101, 194504. 